\title{
Structure and properties of densified silica glass: characterizing the order within disorder
}

\author{
Yohei Onodera ${ }^{1,2}$, Shinji Kohara $\mathbb{1}^{2,3,4,5}$, Philip S. Salmon (10), Akihiko Hirata ${ }^{7,8,9,10}$, Norimasa Nishiyama ${ }^{4,11,12}$, \\ Suguru Kitani ${ }^{11}$, Anita Zeidler $\mathbb{0}^{6}$, Motoki Shiga ${ }^{4,13,14}$, Atsunobu Masuno $\mathbb{1}^{2,15}$, Hiroyuki Inoue ${ }^{16}$, Shuta Tahara ${ }^{2,17}$, \\ Annalisa Polidori $\mathbb{1 0}^{6,18}$, Henry E. Fischer $\mathbb{D}^{18}$, Tatsuya Mori $\mathbb{1 D}^{19}$, Seiji Kojima ${ }^{19}$, Hitoshi Kawaji ${ }^{11}$, \\ Alexander I. Kolesnikov $\mathbb{B}^{20}$, Matthew B. Stone $\mathbb{C}^{20}$, Matthew G. Tucker ${ }^{20}$, Marshall T. McDonnell ${ }^{21}$, Alex C. Hannon ${ }^{22}$, \\ Yasuaki Hiraoka 2,14,23,24, Ippei Obayashi ${ }^{10,14,24}$, Takenobu Nakamura ${ }^{4,25}$, Jaakko Akola ${ }^{26,27}$, Yasuhiro Fujii $\mathbb{C}^{28}$, \\ Koji Ohara $\mathbb{1}^{5}$, Takashi Taniguchi ${ }^{29}$ and Osami Sakata ${ }^{3}$
}

\begin{abstract}
The broken symmetry in the atomic-scale ordering of glassy versus crystalline solids leads to a daunting challenge to provide suitable metrics for describing the order within disorder, especially on length scales beyond the nearest neighbor that are characterized by rich structural complexity. Here, we address this challenge for silica, a canonical network-forming glass, by using hot versus cold compression to (i) systematically increase the structural ordering after densification and (ii) prepare two glasses with the same high-density but contrasting structures. The structure was measured by high-energy X-ray and neutron diffraction, and atomistic models were generated that reproduce the experimental results. The vibrational and thermodynamic properties of the glasses were probed by using inelastic neutron scattering and calorimetry, respectively. Traditional measures of amorphous structures show relatively subtle changes upon compacting the glass. The method of persistent homology identifies, however, distinct features in the network topology that change as the initially open structure of the glass is collapsed. The results for the same highdensity glasses show that the nature of structural disorder does impact the heat capacity and boson peak in the lowfrequency dynamical spectra. Densification is discussed in terms of the loss of locally favored tetrahedral structures comprising oxygen-decorated $\mathrm{SiSi}_{4}$ tetrahedra.
\end{abstract}

\section{Introduction}

Glassy materials often share the same local structural motifs as their crystalline counterparts but feature network topologies that lack translational periodicity ${ }^{1}$. The precise structure of glass is variable because it depends on the interplay between the structural relaxation times and the quenching timescale of the melt. For example, the glass formed by rapid quenching will share structural

\footnotetext{
Correspondence: Shinji Kohara (kohara.shinji@nims.go.jp) or

Philip S. Salmon (p.s.salmon@bath.ac.uk)

${ }^{1}$ Institute for Integrated Radiation and Nuclear Science, Kyoto University, 2-

1010 Asashiro-nishi, Kumatori-cho, Sennan-gun, Osaka 590-0494, Japan

${ }^{2}$ Center for Materials Research by Information Integration (CMI2), Research and

Services Division of Materials Data and Integrated System (MaDIS), National

Institute for Materials Science (NIMS), Ibaraki 305-0047, Japan

Full list of author information is available at the end of the article
}

similarities with the high-temperature melt from which it originates, whereas the glass formed by slow quenching will be more ordered because the system is given more time to relax during its unfulfilled quest to crystallize. If the cooling rate is sufficiently slow, then it is possible to maximize the ordering and minimize the configurational entropy before the onset of the glass transition. Such manipulation of the ordering changes the glass properties, but unlike crystalline solids where symmetry rules, characterizing the nature of this order remains an open challenge. Consequently, there is a structure-versusproperties puzzle in need of a solution for understanding basic issues such as the structural precursors for crystallization in glass $^{2}$ and the mechanisms of 
densification $^{3-5}$ for guiding the development of new technological materials ${ }^{6-8}$.

In this work, we consider silica, an exemplar networkforming material, in which corner-sharing tetrahedral $\mathrm{SiO}_{4}$ motifs can form a multitude of glassy and crystalline structures where chemical ordering is preserved ${ }^{9-13}$. We adopt an approach in which the ordering in the glass is systematically increased by exploiting a temperatureinduced low- to high-density transition under constant pressure and by pushing the processing conditions to the precipice of crystallization. At room temperature and pressures up to $20 \mathrm{GPa}$, an essentially tetrahedral network is maintained ${ }^{14}$, and structural relaxation times will lengthen with increasing pressure because of the reduced volume in which atoms can move. Heat is therefore required to ensure relaxation, so the temperature can be used as a control parameter to explore the potential energy landscape of the densified glass. Within this landscape, a conceptual extreme is provided by the notion of a "perfect glass" with minimal configurational entropy, where the magnitude of the free energy may approach that of the crystalline phase ${ }^{15}$.

The glasses formed under pressure were recovered to ambient conditions to give permanently densified materials $^{6-9,13}$, thus facilitating the experimental exploration of different glass structures along with the associated dynamics and thermodynamics. The glass structure was probed by combining high-energy $\mathrm{X}$-ray diffraction with neutron diffraction, and the dynamical and thermal properties of the material were measured by using inelastic neutron scattering (INS) and calorimetry, respectively. Atomistic structural models were created by using the diffraction data to refine classical molecular dynamics (MD) models via the reverse Monte Carlo (RMC) method ${ }^{16}$. These MD-RMC models were then interrogated using the method of persistent homology ${ }^{17,18}$ to identify and characterize the differences in the network topology that express themselves in the measured diffraction patterns ${ }^{19}$. In the context of material modeling, the aim of persistent homology is to identify the main topological features of the point cloud provided by the positions of the atoms. The method chooses a length scale, identifies the homology of the topological features found on that length scale, and then ascertains how this homology changes as the length scale is varied. Features that persist over the longest range of length scales best capture the underlying topology of the point cloud and hence the material structure.

We have found that persistent homology provides a clear structural signature of the network collapse with increasing density that is not provided by traditional measures of glass structure such as the bond angle, dihedral angle, and ring-size distributions. This method thereby identifies topological features in the structure that likely develop into the nucleation centers for the growth of coesite, the silicon dioxide polymorph into which the glass crystallizes. We also have determined that the highest-density glass has a structure that is different from that of a metastable same-density glass prepared via a cold-compression route, a contrast that leads to dissimilar thermal and low-frequency dynamical properties. This finding is important because recent work has called into question the significance of structural disorder in originating the boson peak, which describes an excess in the vibrational density of states (VDOS) relative to the Debye model $^{20,21}$. Does disorder merely smear out a pile-up of states at the Brillouin zone boundary of the same-density crystal, leading to a small or negligible effect on the heat capacity in a regime where low-temperature tunneling is not observed? Our new results show that the nature of the structural disorder does have a measurable effect on (i) the distribution of low-frequency vibrational modes that contribute toward the boson peak and (ii) the heat capacity.

\section{Materials and methods Glass preparation}

The densified glasses were prepared from fused silica (T-4040, Covalent Materials Corp.) with an $\mathrm{OH}$ concentration of $800 \mathrm{ppm}$. The glasses were designated by their processing conditions, e.g., $1200^{\circ} \mathrm{C} / 7.7 \mathrm{GPa}$ refers to the material recovered after hot compression at $1200^{\circ} \mathrm{C}$ and $7.7 \mathrm{GPa}$. The glasses densified at $7.7 \mathrm{GPa}$ were prepared using a belt-type high-pressure apparatus ${ }^{22}$. The starting glass was in the form of a cylinder (diameter $5.7 \mathrm{~mm}$, thickness $1.5 \mathrm{~mm}$ ) and was enclosed in a capsule made from a tantalum sleeve, tantalum disks, and graphite lids. The glass was compacted under a pressure of 7.7 GPa. For room temperature (RT)/7.7 GPa, this pressure was maintained for 30 min before it was relieved. For the other glasses, the compressed sample was heated from 25 to $300{ }^{\circ} \mathrm{C}$ in $10 \mathrm{~min}$ and from $300{ }^{\circ} \mathrm{C}$ to a target temperature of $400,600,800,1000,1200$ or $1300^{\circ} \mathrm{C}$ in $\sim 20 \mathrm{~s}$, where the power was fine-tuned during the last $10 \mathrm{~s}$ to avoid a temperature overshoot. This target temperature was maintained for $30 \mathrm{~min}$ before the sample was cooled to $300{ }^{\circ} \mathrm{C}$ in $5 \mathrm{~min}$ and from $300^{\circ} \mathrm{C}$ to $\mathrm{RT}$ in $10 \mathrm{~min}$. The pressure was finally relieved. The uncertainty of the pressure of $7.7 \mathrm{GPa}$ at temperatures up to $1250^{\circ} \mathrm{C}$ was $<4 \%{ }^{22}$. The $1300{ }^{\circ} \mathrm{C} / 7.7 \mathrm{GPa}$ sample crystallized by heterogeneous nucleation at the interface with the wall of its capsule. The RT/20 GPa sample was fabricated using a Kawai-type multi-anvil apparatus. A glass sample of diameter $2.5 \mathrm{~mm}$ and thickness $1.8 \mathrm{~mm}$ was embedded in a magnesia pressure medium. The target pressure of $20 \mathrm{GPa}$ was maintained for $2 \mathrm{~h}$ at RT.

The mass density $\rho$ of the as-prepared glassy $\mathrm{SiO}_{2}$ samples was measured using a Micromeritics AccuPycII 
1340 gas pycnometer. Structural relaxation of the densified glasses under ambient conditions was monitored over a time period of $1 \frac{1 / 2}{2}$ years. The densities of the crystalline $\mathrm{SiO}_{2}$ polymorphs were obtained from the lattice parameters measured in neutron diffraction experiments using the Nanoscale-Ordered Materials Diffractometer NOMAD at the Spallation Neutron Source (SNS), Oak Ridge National Laboratory.

\section{High-energy X-ray and neutron diffraction experiments}

The X-ray diffraction experiments were performed in transmission geometry using beamline $\mathrm{BL} 4 \mathrm{~B}^{23}$ at SPring- 8 with an incident energy of $61.4 \mathrm{keV}$. The incident X-ray intensity was monitored using an Ar-filled ionization chamber, and the scattered $\mathrm{X}$-rays were detected by a solid-state Ge detector. A vacuum chamber was used to suppress air scattering, and the data were corrected using a standard program ${ }^{23}$. Each data set was normalized to give the Faber-Ziman total structure factor

$$
S(Q)=1+\frac{1}{|\langle w(Q)\rangle|^{2}} \sum_{\alpha} \sum_{\beta} c_{\alpha} c_{\beta} w_{\alpha}^{*}(Q) w_{\beta}(Q)\left[S_{\alpha \beta}(Q)-1\right],
$$

where $Q$ is the magnitude of the scattering vector, $c_{\alpha}$ is the atomic fraction of chemical species $\alpha$ ( $\mathrm{Si}$ or $\mathrm{O}), w_{\alpha}(Q)$ is the atomic form factor with dispersion terms for chemical species $\alpha, S_{\alpha \beta}(Q)$ is the partial structure factor for chemical species $\alpha$ and $\beta, \quad$ and $|\langle w(Q)\rangle|^{2}=\sum_{\alpha} \sum_{\beta} c_{\alpha} c_{\beta} w_{\alpha}^{*}(Q) w_{\beta}(Q)$. The total correlation function $T(r)$ was obtained from the Fourier transform relation

$$
T(r)=4 \pi n_{0} r+\frac{2}{\pi} \int_{0}^{\infty} \mathrm{d} Q[S(Q)-1] M(Q) Q \sin (Q r),
$$

where $r$ is the distance in real space and $n_{0}$ is the atomic number density. $M(Q)$ is the Lorch modification function given by $M(Q)=\sin \left(\pi Q / Q_{\max }\right) /\left(\pi Q / Q_{\max }\right)$ for $Q \leq Q_{\max }$ and $M(Q)=0$ for $Q>Q_{\max }$, where $Q_{\max }$ is the maximum value of $Q$.

The neutron diffraction experiment for RT/20 GPa was performed using the General Materials diffractometer GEM at the ISIS spallation source ${ }^{24}$, and the neutron diffraction experiments on the other samples were performed using the D4c diffractometer at the Institut LaueLangevin (ILL) ${ }^{25}$. Measurements were made for each sample held in a vanadium container, the empty container, the empty instrument, and a vanadium-5.14\% niobium or vanadium standard for normalization purposes. The neutron total structure factors $S(Q)$ are defined by Eq. (1), but with $w_{\alpha}(Q)$ set equal to the $Q$-independent coherent neutron scattering length of chemical species $\alpha$, and were obtained by following a standard data analysis procedure. The total correlation functions $T(r)$ were obtained from Eq. (2).

\section{INS experiments}

The INS experiments were performed using the fine resolution Fermi chopper spectrometer SEQUOIA ${ }^{26,27}$ at the SNS. The mass of each sample was, in general, small (Table S1). The samples were held in thin aluminum foil, and the measurements were performed at room temperature $(T=294 \mathrm{~K})$. To provide high-energy resolution at low energies, $\sim 1 \mathrm{meV}$ at the elastic line and better at higher energy transfers, the data were collected with an incident neutron energy $E_{i}=35 \mathrm{meV}$. The $Q$ range covered in the experiments was $0.2-3.8 \AA^{-1}$. The INS spectra for the empty aluminum foil were measured under the same conditions and subtracted from the sample data. The INS spectra for the vanadium standard were also measured using a "white" incident neutron beam to normalize the detector efficiencies. The collected INS data were corrected for the detector efficiency and transformed from the time-of-flight and instrument coordinates to the dynamical structure factor $S(Q, E)$, where $E$ is the energy transfer, by using the MANTIDPLOT ${ }^{28}$ and DAVE ${ }^{29}$ software packages for data reduction and analysis.

\section{Specific heat capacity measurements}

The specific heat capacity at constant pressure $c_{P}$ was measured in the temperature range of $1.8-310 \mathrm{~K}$ by a relaxation technique using a Quantum Design Physical Property Measurement System. The sample was attached to the instrument platform with Apiezon $\mathrm{N}$ grease. For each measurement, the addendum (platform and thermal grease) heat capacity was measured separately and subtracted.

\section{MD-RMC models of the glass structure}

The MD calculations were performed for a system of 3000 atoms (1000 Si and $2000 \mathrm{O}$ ) using an in-house code within the $N V T$ ensemble. The interactions were described by pair potentials with short-range Born-Mayer repulsive and long-range Coulomb terms, i.e.,

$$
\phi_{i j}(r)=B_{i j} \exp \left(-\frac{r}{\rho_{i j}}\right)+\frac{e^{2}}{4 \pi \varepsilon_{0}} \frac{Z_{i} Z_{j}}{r},
$$

where $r$ is the distance between atoms $i$ and $j, B_{i j}$ and $\rho_{i j}$ define the magnitude and softness of the Born-Mayer term, respectively, $Z_{i}$ is the effective charge on atom $i$ $\left(Z_{\mathrm{Si}}=2.4, Z_{\mathrm{O}}=-1.2\right), e$ is the elementary charge, and $\varepsilon_{0}$ is the permittivity of vacuum. The $B_{i j}$ values were $21.39 \times$ $10^{-16} \mathrm{~J}(\mathrm{Si}-\mathrm{O}), 0.6246 \times 10^{-16} \mathrm{~J}(\mathrm{O}-\mathrm{O})$ or zero $(\mathrm{Si}-\mathrm{Si})$; the $\rho_{i j}$ values were $0.174 \AA(\mathrm{Si}-\mathrm{O}), 0.362 \AA(\mathrm{O}-\mathrm{O})$ or zero $(\mathrm{Si}-\mathrm{Si})$. The simulations used periodic boundary 
conditions, and the long-range Coulomb interactions were treated using the Ewald summation. A time step of $1 \mathrm{fs}$ was used in the Verlet algorithm.

Four different structural models were obtained for $\mathrm{SiO}_{2}$ glass at ambient pressure from different random starting configurations by equilibrating at $4000 \mathrm{~K}$ for 20,000 timesteps, cooling to $293 \mathrm{~K}$ during 200,000 time-steps, and annealing at $293 \mathrm{~K}$ for 20,000 time-steps. These models were used as starting points for modeling the structures of four different densified glasses. For RT/7.7 GPa and RT/ $20 \mathrm{GPa}$, the volume of the unit cell was shrunk step-bystep during 100,000 time-steps to give the measured density at a pressure of 7.7 or $20 \mathrm{GPa}$, respectively. For $400{ }^{\circ} \mathrm{C} / 7.7 \mathrm{GPa}$ and $1200{ }^{\circ} \mathrm{C} / 7.7 \mathrm{GPa}$, the system was heated to 673 or $1473 \mathrm{~K}$ at a rate of 0.1 or $0.3 \mathrm{~K}$ per 25 time-steps, respectively. The volume of the unit cell was then shrunk step-by-step during 100,000 time-steps to give the measured density for a pressure of 7.7 GPa. Finally, the temperature was lowered to $293 \mathrm{~K}$ during 10,000 time-steps, and the system was annealed at $293 \mathrm{~K}$ for 20,000 time-steps.

The MD atomic configurations were refined by using the $\mathrm{RMC}++\operatorname{code}^{30}$. In these RMC refinements, the MD results for the $\mathrm{Si}-\mathrm{O}$ coordination number distributions and intra-tetrahedral $\mathrm{O}-\mathrm{Si}-\mathrm{O}$ bond angle distributions were used as constraints.

\section{Measures of the glass structure}

A cavity volume analysis of the MD-RMC models for glassy $\mathrm{SiO}_{2}$ was performed using the pyMolDyn code ${ }^{31}$. In the calculation of the bond angle distribution $B(\theta)$, the values are proportional to the number of bonds between $\theta$ and $\theta+\Delta \theta$, which is dependent on the solid angle $\Delta \Omega \propto$ $\sin (\theta)$ subtended at that value of $\theta$. Each bond angle distribution was therefore plotted as $B(\theta) / \sin (\theta)$ to compensate for the effect of $\Delta \Omega$. In this way, a finite bond angle distribution at $\theta \simeq 180^{\circ}$ will not be artificially suppressed $^{32}$. The dihedral angle and primitive ring-size distributions were calculated using the R.I.N.G.S. $\operatorname{code}^{33}$. Primitive rings are identified by making a shortest closedpath search along with $\mathrm{Si}-\mathrm{O}$ bonds and cannot be decomposed into smaller rings ${ }^{34}$.

\section{Persistent homology analysis}

Persistent homology ${ }^{17,18}$ was used to characterize the topology of the densified glasses and crystalline polymorphs. The principles of this method are outlined below, and the analysis was performed by using the HomCloud package $^{35}$. Optimal cycles were identified, which correspond to the minimal path lengths around a hole ${ }^{36}$.

Persistent homology is an emerging tool in topological analysis for capturing the shape of data sets in a multiscale manner. For the atomistic models of materials, the data set is provided by a point cloud defined by the atomic coordinates, and the aim of persistent homology is to find the main topological features of this set of points. These features can be identified by centering a sphere of radius $r(\alpha)=\sqrt{\alpha}$ on each atom of either Si or O. As the radius is grown uniformly from zero by increasing the parameter $\alpha$, the topology of the space defined by the union of all spheres will change. For instance, several of the initially isolated spheres could merge to form a cluster that (i) does not enclose a hole, (ii) encloses a 2D hole, or (iii) encloses a 3D cavity, where these scenarios belong to the 0th, 1st, and 2nd homology classes, respectively. The homology class is identified by forming a closed path or cycle between the centers of the spheres and inspecting the space enclosed.

For open silica-like networks, cycles from the 1st homology class are the most informative ${ }^{17}$. Here, the birth of a cycle is marked by the radius at which the spheres first overlap to enclose a hole. As the sphere radius grows, the cycle will persist until the enclosed hole is covered by overlapping spheres. The corresponding sphere radius marks the death of the cycle when the 1st homology information is lost. Eventually, for a sufficiently large radius, the spheres will merge to become a single connected space that contains no holes (or cavities).

The results from persistent homology can be visualized in the form of a persistence diagram (PD). Let $c_{i j}$ be the cycle that is created when $\alpha=b_{i}$ and eliminated when the hole is covered at $\alpha=d_{j}$. The parameters $b_{i}$ and $d_{j}$ are referred to as the birth and death scales for $c_{i j}$ and are measures of the maximum distance between two adjacent atoms in a cycle and the overall size of that cycle, respectively. The multiplicity $M_{i j}$ of $c_{i j}$ for each pair $\left(b_{i}, d_{j}\right)$ is logged, and the results can be presented as a PD, which is a two-dimensional histogram. For a given cycle, it follows that $b_{i}<d_{j}$, so features in a PD will appear above the birth $=$ death diagonal.

As the sphere radius is grown, longer length scales are probed. The most persistent cycles are the longest lived; i.e., they have the largest difference between the birth and death scales. Persistent cycles can therefore exist across multiple length scales and are the most representative of the system's topology. For an atomic network, these cycles are born when the sphere radius $r\left(b_{i}\right)=\sqrt{b_{i}} \approx r_{0} / 2$, where $r_{0}$ is the mean nearest-neighbor distance. In a PD, persistent cycles therefore appear with $b_{i}^{2} \approx r_{0}^{2} / 4$ and extend to large death values. Once a persistent cycle is born on a given length scale, secondary cycles can be generated from this cycle as the length scale is grown. These secondary cycles are more transient than persistent cycles; i.e., they have similar birth and death scales and therefore appear near the birth = death diagonal in a PD. A schematic illustrating persistent versus transient cycles is shown in Fig. S1.

It should be noted that cycles are constructed in a different way to primitive rings where a closed path is traced 
by following the atomic bonds ${ }^{34}$. In general, persistent cycles and primitive rings will therefore deliver different information on the atomic arrangements.

\section{Tetrahedral order parameter}

In the glass structure, oxygen-decorated $\mathrm{SiSi}_{4}$ tetrahedra can be regarded as locally favored tetrahedral structures (LFTSs). The tetrahedral order parameter for these Sicentered tetrahedra is defined by ${ }^{37}$

$$
q \equiv 1-\frac{3}{8} \sum_{i=1}^{3} \sum_{k=i+1}^{4}\left(\frac{1}{3}+\cos \theta_{i j k}\right)^{2},
$$

where $\theta_{i j k}$ is the angle formed between the central Si atom $j$ and its neighboring Si atoms $i$ and $k$. This parameter was designed to give unity for a regular tetrahedron and a mean value of zero for a perfect gas.

\section{Results}

Permanently densified silica glasses were synthesized by hot compression at a pressure of $7.7 \mathrm{GPa}$ and temperatures up to $1200^{\circ} \mathrm{C}$, beyond which the glass crystallizes to coesite. Permanently densified glass was also made by cold compression at a pressure of $20 \mathrm{GPa}$ and room temperature (RT). The glasses were stable for at least $1 \frac{1 / 2}{2}$ years under ambient conditions, except RT/20 GPa, which showed a reduction in density of $2.8 \%$ on this timescale (Table S2).

The experimental results for the glasses densified at 7.7 GPa indicate the transformation from a low- to highdensity amorphous phase for a processing temperature of $\sim 600^{\circ} \mathrm{C}$. There is a clear alteration to the rate-of-change of the density with processing temperature (Fig. 1A) that is accompanied by a marked change in the sharpness of the first sharp diffraction peak (FSDP) in the total structure factor $S(Q)$ measured in the diffraction work ${ }^{38}$, which appears at a scattering vector $Q_{\mathrm{FSDP}} \sim 1.5 \AA^{-1}$ for RT/ 7.7 GPa (Fig. 1B). The corresponding $T(r)$ functions (Fig. $\mathrm{S} 2$ ) give a nearest-neighbor $\mathrm{Si}-\mathrm{O}$ coordination number of four within the experimental error (Table S3); i.e., the structure of each glass is based on a network of cornersharing tetrahedral $\mathrm{SiO}_{4}$ motifs. The correlation length describing the ordering of these motifs on an intermediate length scale is given by $2 \pi / \Delta Q_{\mathrm{FSDP}}$, where $\Delta Q_{\mathrm{FSDP}}$ is the full width at half-maximum of the FSDP. For the glasses compacted at $7.7 \mathrm{GPa}$, the correlation length first decreases and then increases with density, reaching a maximum of $12.7(4) \AA$ for $1200^{\circ} \mathrm{C} / 7.7 \mathrm{GPa}$ (Fig. 1C), just before the onset of crystallization. The density of both the $1200{ }^{\circ} \mathrm{C} / 7.7 \mathrm{GPa}$ and $\mathrm{RT} / 20 \mathrm{GPa}$ glasses is $\sim 23 \%$ larger than the pristine glass, but the glass structures are notably different (Fig. 1B), with a correlation length for $1200^{\circ} \mathrm{C} /$ 7.7 GPa that is $\sim 60 \%$ longer than for RT/20 GPa (Fig. 1C).
Structural differences on an intermediate length scale are often discussed in terms of the low-frequency dynamics of a glass network ${ }^{39-41}$. INS experiments were therefore performed to observe the behavior of the boson peak. Contour maps of the measured dynamical structure factors are shown in Fig. 2, and sections through these $S(Q, E)$ functions are shown in Fig. 3A. The effective total VDOS obtained from each $S(Q, E)$ function ${ }^{42,43}$ is shown in Fig. S3. The temperature dependence of the constantpressure specific heat capacity $c_{P}$ was also measured because the excess in VDOS leading to the boson peak reveals itself as a peak positioned at $T_{\max }$ in a plot of $c_{P} / T^{3}$ versus the absolute temperature $T$ (Fig. $3 \mathrm{~B})^{44}$. The measured heat capacities are consistent with previous work (Fig. S4). Both sets of measurements show a response to the transformation from a low- to high-density amorphous phase for the glasses densified at $7.7 \mathrm{GPa}$. The density dependence of the boson peak position $E_{\mathrm{BP}}$ for $1200^{\circ} \mathrm{C} / 7.7 \mathrm{GPa}$ is not colinear with the densitydependent positions for $\mathrm{RT} / 7.7 \mathrm{GPa}$ and $400^{\circ} \mathrm{C} / 7.7 \mathrm{GPa}$ (Fig. 3C), and $T_{\max }$ shows superlinear behavior for a processing temperature $\geq 600^{\circ} \mathrm{C}$ (Fig. 3D). The INS spectra do not reveal a contribution to the boson peak from excitations on a length scale associated with the FSDP (Fig. 2).

The densities of $1200^{\circ} \mathrm{C} / 7.7 \mathrm{GPa}$ and $\mathrm{RT} / 20 \mathrm{GPa}$ are comparable and similar to the density of crystalline $\alpha$ quartz (Fig. 1A). Chumakov et al. ${ }^{20,21}$ reported that many of the differences in the low-frequency VDOS and specific heats between $\mathrm{SiO}_{2}$ glasses and crystals, which have been attributed to structural disorder, can be reconciled by matching the glass density with that of a crystalline polymorph. Accordingly, the heat capacities for $1200^{\circ} \mathrm{C} /$ 7.7 GPa and RT/20 GPa are comparable and similar to the heat capacity for $\alpha$-quartz (Fig. 3B). However, closer inspection of the results shows a shift in $T_{\max }$ (Fig. 3D). The boson peak for $1200^{\circ} \mathrm{C} / 7.7 \mathrm{GPa}$ is also displaced towards a larger $E_{\mathrm{BP}}$ value relative to $\mathrm{RT} / 20 \mathrm{GPa}$, with a profile that is broader and stretched towards higher energies (Fig. 3A, C). Hence, the nature of the glass structure, as expressed by the coordination environments of tetrahedral $\mathrm{SiO}_{4}$ motifs, is also important for understanding the origin of the boson peak.

\section{Characterizing the glass structure}

To investigate the mechanisms of structural collapse and the extent of structural similarity between the glassy and crystalline forms of $\mathrm{SiO}_{2}$, the diffraction data were used to prepare MD-RMC models for the glass structures that reproduce the measured X-ray and neutron total structure factors (Fig. S5). The corresponding partial structure factors are shown in Fig. S6. For the glasses densified at $7.7 \mathrm{GPa}$, the models show a marked reduction in size and the removal of connected regions of free 

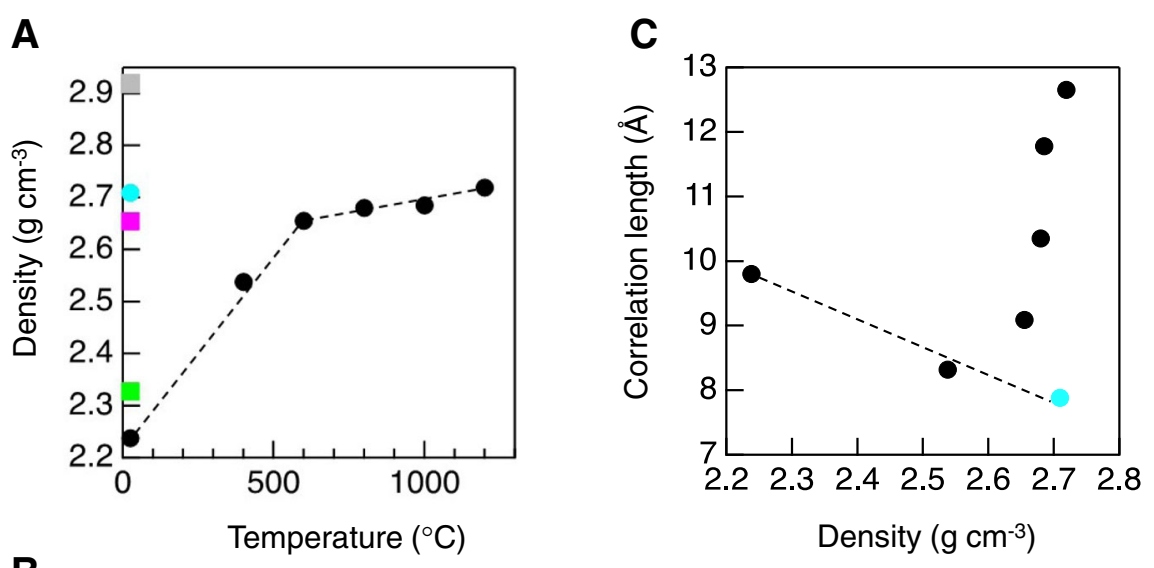

B

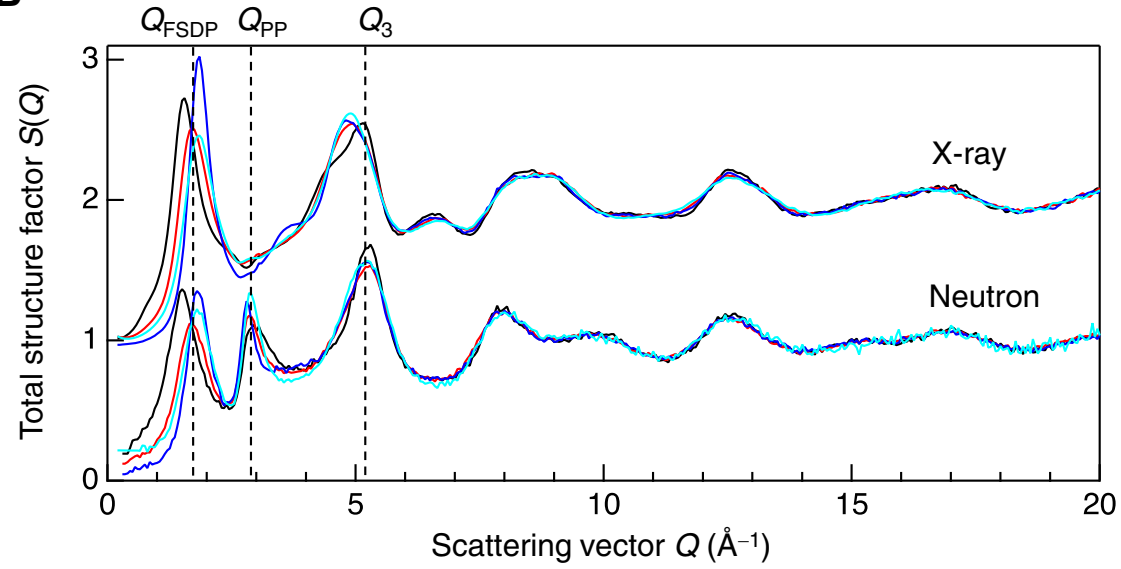

Fig. 1 Comparison between the densities and structures of glassy and crystalline $\mathbf{S i O}_{\mathbf{2}}$. A Density of the glasses compacted at $7.7 \mathrm{GPa}$ and different temperatures (black circles), RT/20 GPa (cyan circle), a-cristobalite (green square), a-quartz (magenta square) and coesite (gray square). For the glasses compacted at $7.7 \mathrm{GPa}$, the straight lines are drawn as guides to the eye, and their intersection indicates a change in behavior at a temperature of $\sim 600^{\circ} \mathrm{C}$. The hot-compressed glass crystallized into coesite at a temperature $>1200^{\circ} \mathrm{C}$. B The X-ray and neutron $S(Q)$ functions for RT/ 7.7 GPa (black), $400^{\circ} \mathrm{C} / 7.7 \mathrm{GPa}$ (red), $1200^{\circ} \mathrm{C} / 7.7 \mathrm{GPa}$ (blue) and RT/20 GPa (cyan). The vertical broken lines mark the approximate positions of the FSDP at $Q_{F S D P}$, the principal peak at $Q_{P p}$ and the third peak at $Q_{3}$. C The density dependence of the correlation length $2 \pi / \triangle Q_{F S D P}$ extracted from the X-ray total structure factors for the glasses compacted at $7.7 \mathrm{GPa}$ and different temperatures (black circles) versus RT/20 GPa (cyan circle). For the glasses compacted at $7.7 \mathrm{GPa}$, a change to the density dependence of the correlation length is shown by a deviation from the straight line at a density $>2.54 \mathrm{~g} \mathrm{~cm}^{-3}$, corresponding to a processing temperature between 400 and $600{ }^{\circ} \mathrm{C}$. The error bars are smaller than the symbol size or line thickness.

volume with increased processing temperature; i.e., the low- to high-density amorphous transition is accompanied by a striking reduction in cavity volume (Fig. 4).

To extract information on the 3D atomic configurations, we performed a traditional analysis to calculate the bond angle, dihedral angle and ring-size distributions. Changes to the bond angle (Fig. 5A) and dihedral angle (Fig. 5B) distributions are most noticeable when progressing from $\mathrm{RT} / 7.7 \mathrm{GPa}$ to $400^{\circ} \mathrm{C} / 7.7 \mathrm{GPa}$ but are small for a further increase in the glass density. For the coldcompressed glasses, the shift in the $\mathrm{Si}-\mathrm{O}-\mathrm{Si}$ bond angle distribution towards a smaller angle and the broadening of this distribution with increasing density is consistent with the results obtained from nuclear magnetic resonance (NMR) spectroscopy experiments ${ }^{13}$. For these glasses, the $\mathrm{Si}-\mathrm{O}$ bond length increases from 1.611(5) $\AA$ for RT/7.7 GPa to 1.616(5) ^ for RT/20 GPa (Table S3), which is also consistent with the NMR results that show an increased $\mathrm{Si}-\mathrm{O}$ bond length with decreased $\mathrm{Si}-\mathrm{O}-\mathrm{Si}$ bond angle ${ }^{13}$. The ring-size distributions show subtle changes between the different glasses (Fig. 6); i.e., these distributions are largely insensitive to the structural changes on an intermediate length scale that manifest themselves by a sharpening of the FSDP (Fig. 1B, C). It is therefore necessary to find an alternative approach to help pick apart differences between network structures. To this end, we used persistent homology as a characterization tool $^{17,18}$.

In each MD-RMC model, the structure can be regarded as an amorphous silicon network that is decorated by 

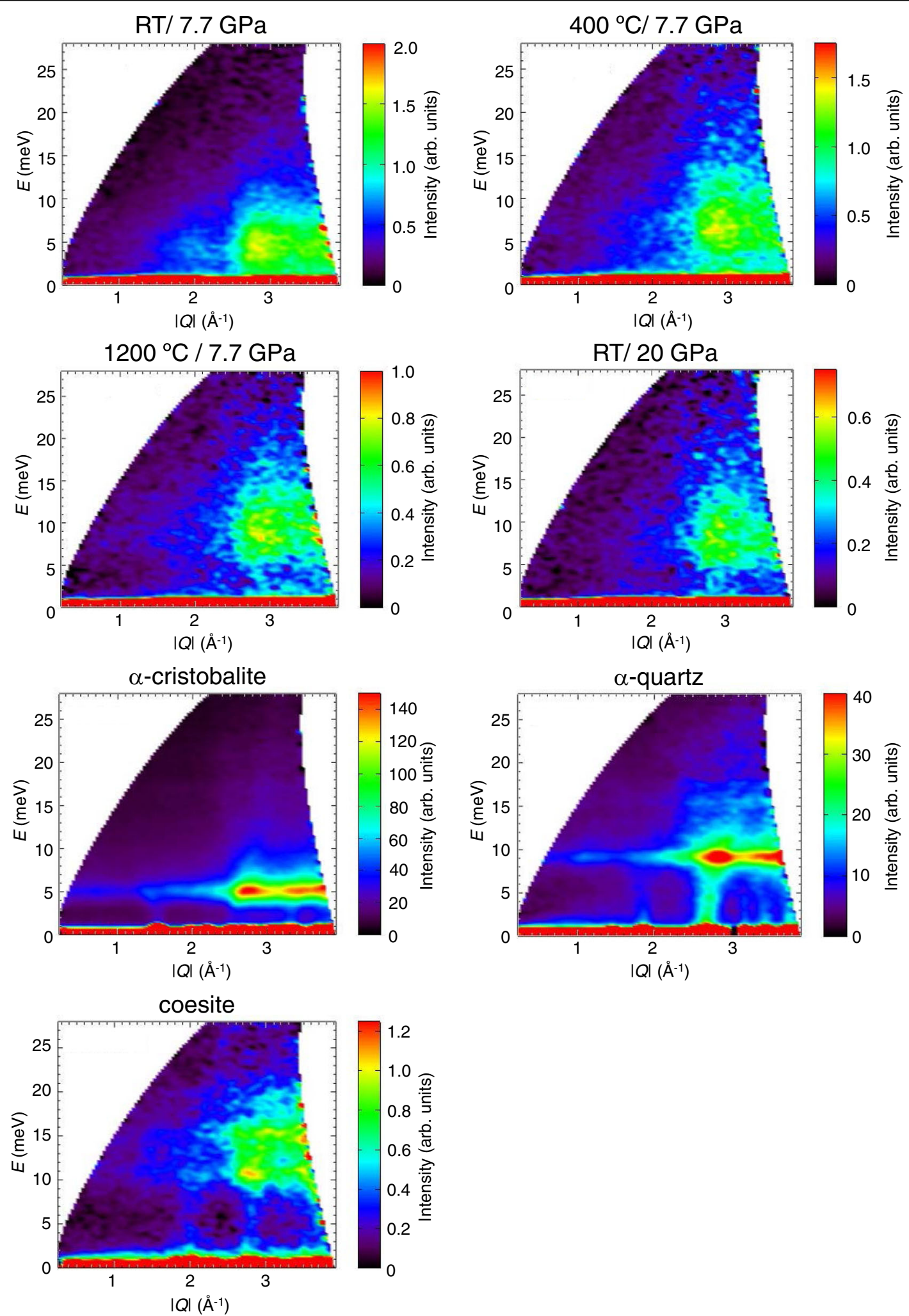

Fig. 2 Contour maps of the dynamical structure factor $S(Q, E)$ measured by INS for glassy and crystalline $\mathrm{SiO}_{2}$. For the glasses, the boson peak is positioned at an energy $E_{\mathrm{BP}}$ in the range $4.6-8.6 \mathrm{meV}$ that depends on the density. There is no contribution toward the boson peak from scattering in the region of the FSDP in the neutron $S(Q)$ function at $Q_{F S D P} \sim 1.50-1.87 \AA^{-1}$ (Fig. 1B) nor from scattering at $Q_{F S D P} / 2$. Instead, the main contributions towards $S(Q, E)$ originate from $Q$-values associated with the principal peak at $Q_{p p} \sim 2.81-2.98 \AA^{-1}$. 

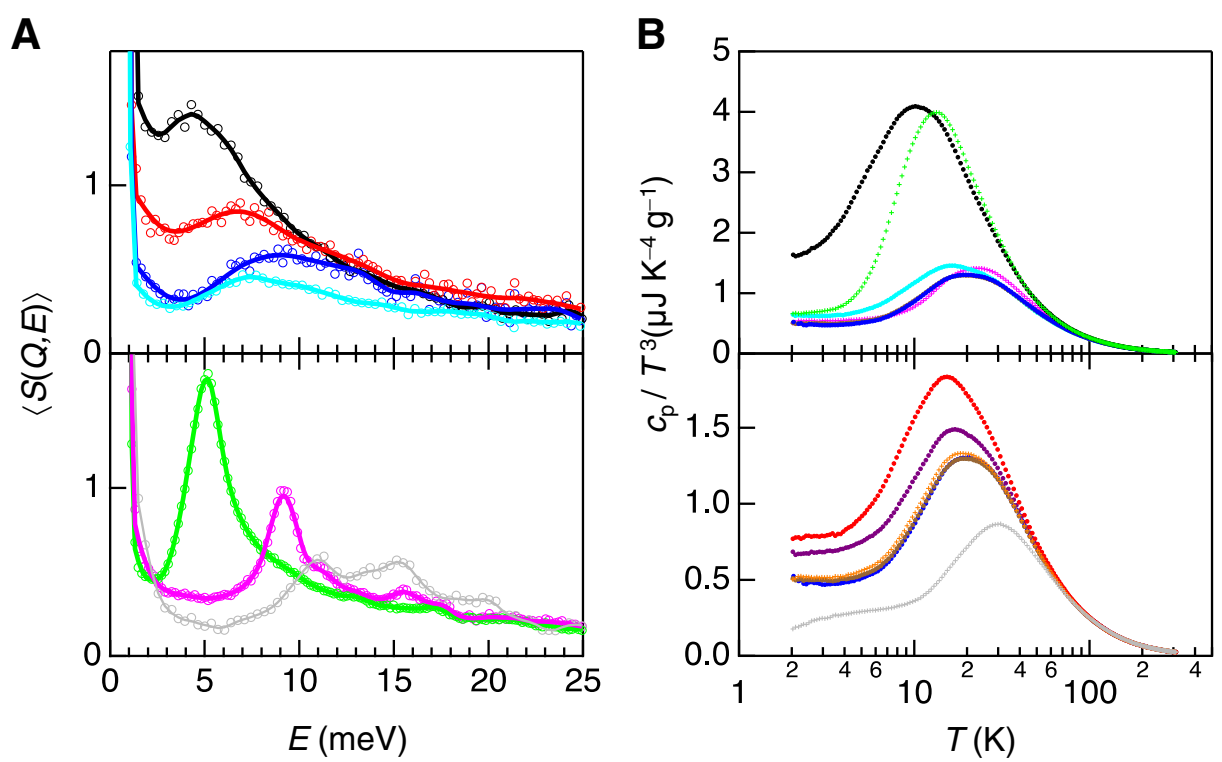

C

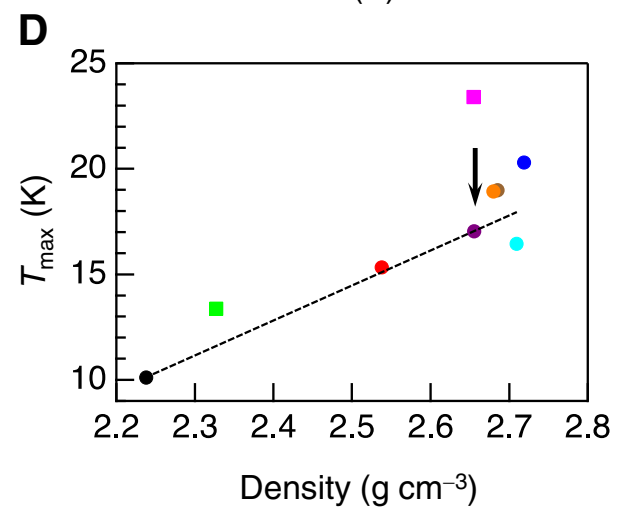

Fig. 3 Comparison between the INS and specific heat capacity results for glassy and crystalline $\mathbf{S i O}_{2}$. A The boson peak region of the dynamical structure factor $\langle S(Q, E)\rangle=\int_{Q_{\min }}^{Q_{\max }} \mathrm{d} Q S(Q, E) /\left(Q_{\max }-Q_{\min }\right)$ measured by INS for (top panel) densified glasses and (bottom panel) crystalline solids where $Q_{\min }=0.4 \AA^{-1}$ and $Q_{\max }=3.6 \AA^{-1}$. B The dependence of $c_{p} / T^{3}$ on temperature $T$ for (top panel) $a$-cristobalite versus a similar density glass, a-quartz versus two similar density glasses, and (bottom panel) coesite versus the other densified glasses. $\mathbf{C}$, $\mathbf{D}$ The density dependence of the boson peak position $E_{\mathrm{BP}}$ shown in $(\mathbf{A})$ and the peak maximum position $T_{\max }$ shown in (B), respectively. In $(\mathbf{A})$ and $(\mathbf{C})$, the curves or symbols represent RT/7.7 GPa (black), $400^{\circ} \mathrm{C} / 7.7 \mathrm{GPa}$ (red), $1200^{\circ} \mathrm{C} / 7.7 \mathrm{GPa}$ (blue), RT/20 GPa (cyan), a-cristobalite (green), a-quartz (magenta) and coesite (gray). These designations also hold for (B) and (D), where additional data points are given for $600^{\circ} \mathrm{C} / 7.7 \mathrm{GPa}$ (purple), $800^{\circ} \mathrm{C} / 7.7 \mathrm{GPa}$ (orange) and $1000^{\circ} \mathrm{C} / 7.7 \mathrm{GPa}$ (brown). In (C) and (D), the data points for coesite (first peak at $E_{\mathrm{BP}}=11 \mathrm{meV}$ and $T_{\max }=31 \mathrm{~K}$ ) are omitted for clarity of presentation. The vertical arrows indicate a change at $\sim 2.66 \mathrm{~g} \mathrm{~cm}^{-3}$ to the density dependence of $E_{\mathrm{BP}}$ and $T_{\max }$ for the glasses compacted at $7.7 \mathrm{GPa}$, which corresponds to a processing temperature of $\sim 600^{\circ} \mathrm{C}$. Error bars are comparable to the symbol size or line thickness.

twofold-coordinated oxygen atoms. The main features in the network topology are, therefore, best expressed in terms of the point cloud formed by the silicon atom coordinates. Figure 7A-D compares the resultant Sicentric PDs for the densified glasses. The boxed regions parallel to the death axis highlight the persistent cycles, which exist across multiple length scales and are the most robust features of the system topology. Persistent cycles are, therefore, the most informative for identifying topological change. The PDs of Fig. 7A-D show a reduction in the death value of the persistent cycles with increasing density.
Figure 7E-H shows three-dimensional representations of the PDs in the region of the persistent cycles. For the hot-compressed glasses in Fig. 7E-G, there is a marked sharpening in the distribution of multiplicities with increased processing temperature. Also, the $1200^{\circ} \mathrm{C} /$ 7.7 GPa and RT/20 GPa glasses have markedly different PDs, as illustrated in Fig. $7 \mathrm{G}$ and H, pointing to a contrast in the network structures that manifests itself in the dynamics and thermodynamics of these same-density materials, as shown in Figs. 2 and 3. Figure 8A shows the Si-centric PDs for the crystalline polymorphs, calculated in the absence of thermal effects that would broaden the 


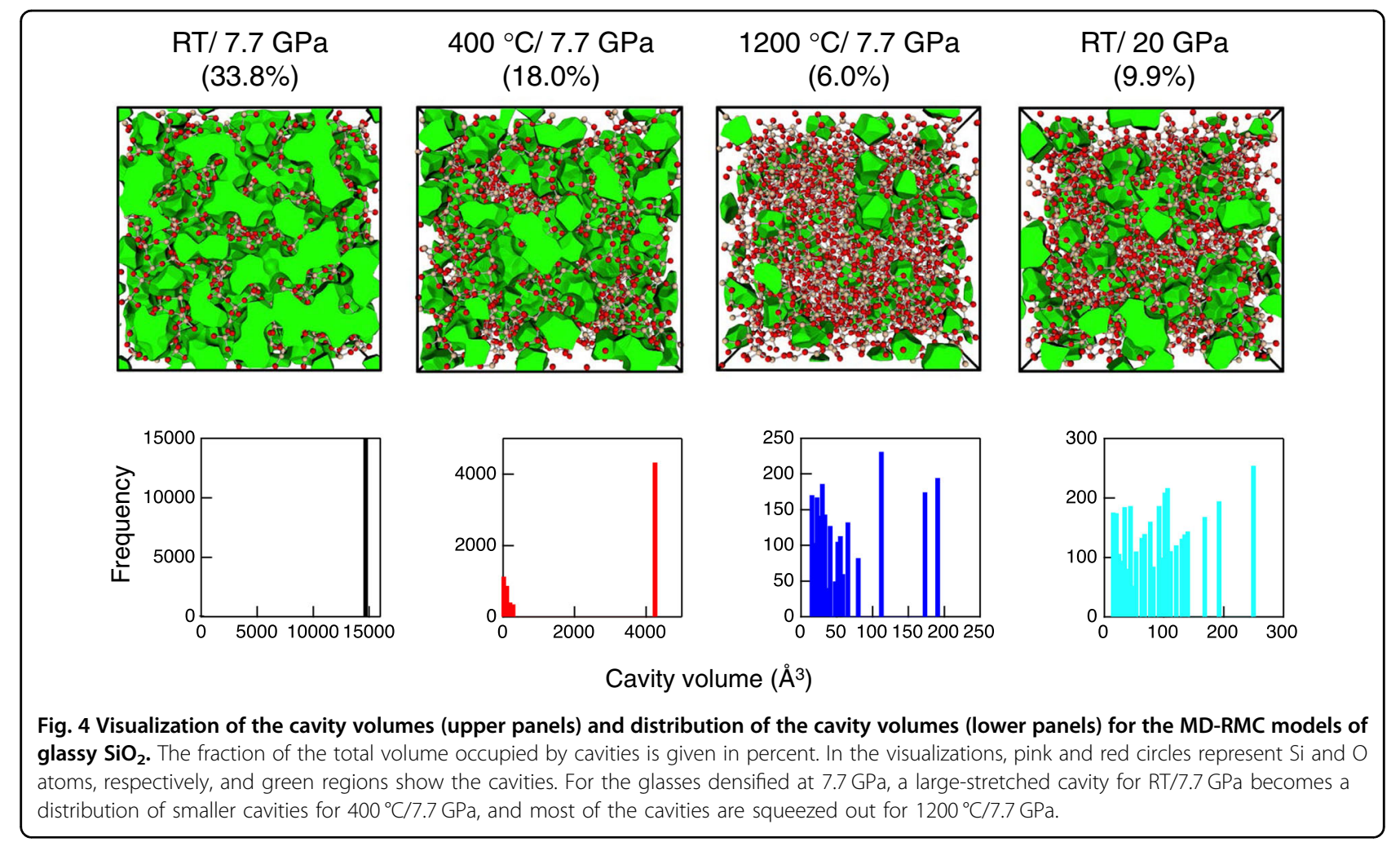

observed distributions. For completeness, the oxygencentric PDs are shown in Fig. S7.

Figure $8 \mathrm{~B}$ compares the multiplicity profiles for the persistent cycles of the glasses in Fig. 7A-D and crystalline polymorphs in Fig. 8A. The profiles for the crystals are represented by bars that fall, for each crystal structure, into regions with clearly delineated $d_{k}$ values, with a maximum death value that reduces as the density increases. For the hot-compressed glasses, the development of a sharp peak in the Si-centric PDs (Fig. 7E-G) is accompanied by a loss of the long-death tail in the multiplicity profile (Fig. 8B), moving the distribution closer to that found for coesite. The birth coordinates of the persistent cycles are similar for each of the materials, so a reduction in the lifetime values $\ell_{i j} \equiv d_{j}-b_{i}$ is anticipated.

The spatial coordinates of the atoms in a persistent cycle will reveal the geometric shape of that cycle, which will be denoted by $N S i$, where $N$ is the number of $\mathrm{Si}$ atoms. In this way, features in the PD can be identified with configurations that describe the network topology. Figure $8 \mathrm{C}$ compares the average lifetime $\left\langle\ell_{i j}(N)\right\rangle$ of the persistent cycles found for each value of $N$ in the crystalline and glassy materials. Several of the associated configurations for the densified glasses are shown in Fig. 9. The results show that unless they are small $(N \leq 5)$, persistent cycles become shorter lived with increasing density, which reflects a change to their geometry. For example, in the case of the glasses compressed at 7.7 GPa, the $\left\langle\ell_{i j}(N)\right\rangle$ values for $N>5$ move closer to those of coesite, the phase into which the glass crystallizes at $\sim 1300^{\circ} \mathrm{C}$. The reduced lifetime values therefore capture the reorganization of the Si atoms in the network as it is compacted, and the cavities are squeezed out (Fig. 4).

\section{Discussion}

The structural transformation strategy that we have adopted takes advantage of a strong-to-fragile transition in silica under cold compression ${ }^{45}$ that moves the glass to a more jagged region of the energy landscape ${ }^{46}$. At room temperature, increased pressure eventually leads to the formation of a metastable glass at $20 \mathrm{GPa}$ with a density that diminishes with time, which suggests access to a region of the landscape in which minima are separated by reduced energy barriers, thus enabling relaxation. At a pressure of $7.7 \mathrm{GPa}$, increased temperature enables more of the jagged landscape to be sampled, pushing the material towards a structure that may approach that of a perfectly ordered glass. The hot versus cold compression routes therefore offer a means of exploring the energy landscapes of network-forming glasses and the transformations thereon. The methodology differs from previous attempts to realize a perfectly ordered network-forming glass, which have relied on the temperature- or pressureinduced amorphization of a crystal structure because of 
A
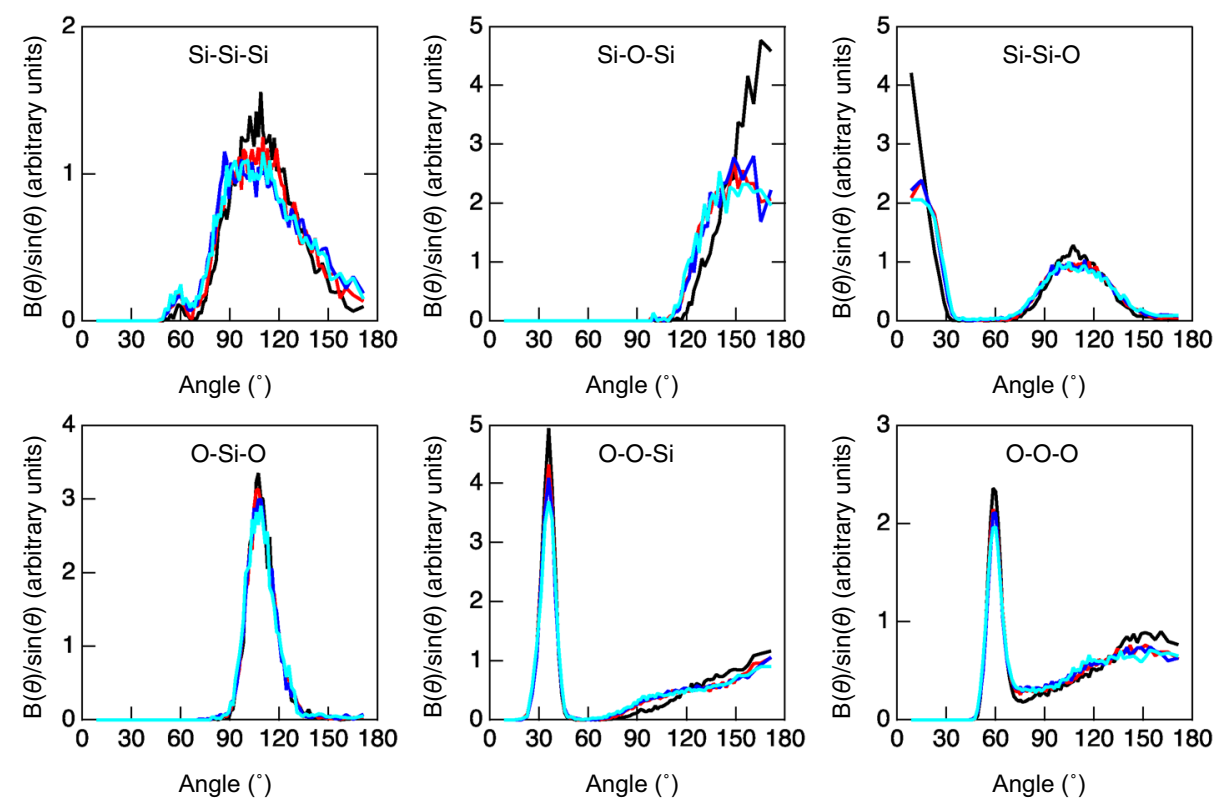

B

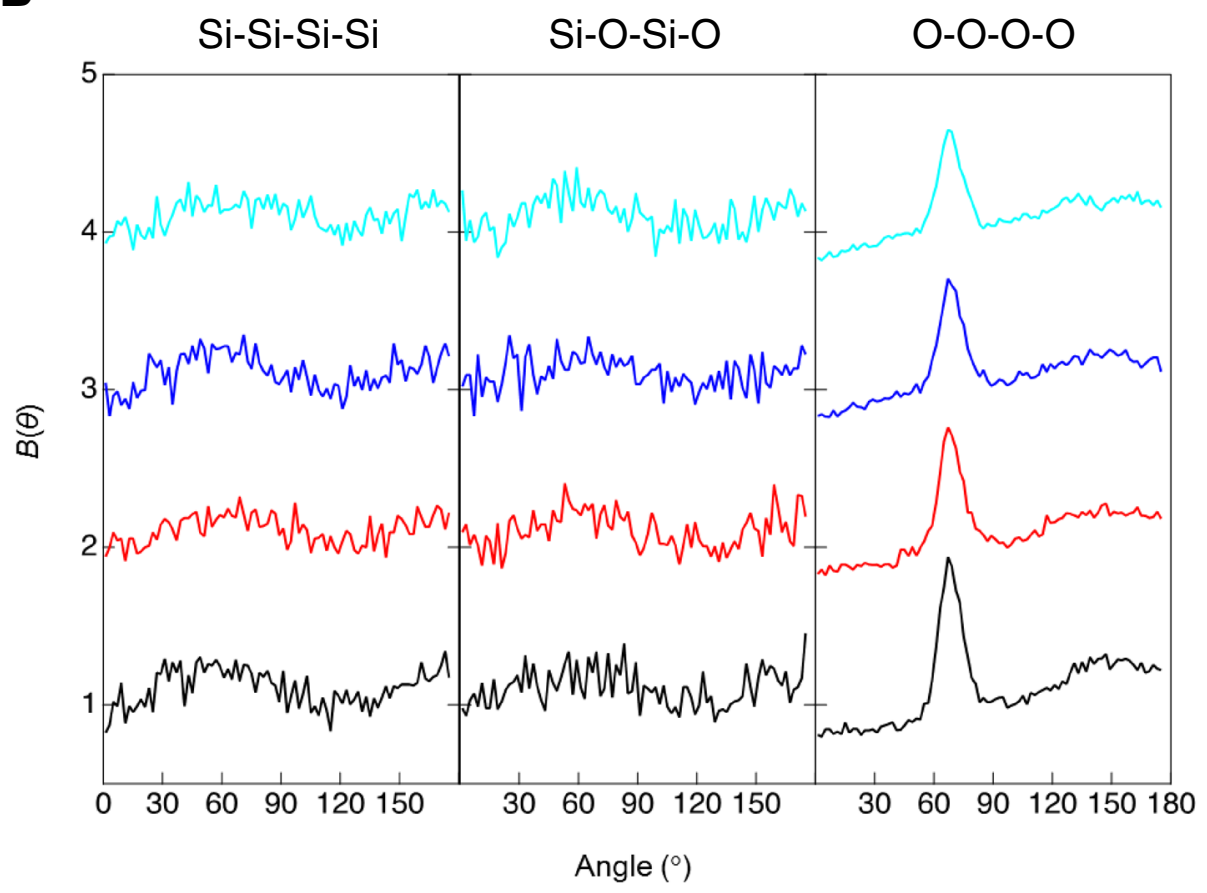

Fig. 5 Bond and dihedral angle distributions for the MD-RMC models of glassy $\mathrm{SiO}_{2}$. A The $\mathrm{Si}-\mathrm{Si}-\mathrm{Si}, \mathrm{Si}-\mathrm{O}-\mathrm{Si}, \mathrm{Si}-\mathrm{Si}-\mathrm{O}, \mathrm{O}-\mathrm{Si}-\mathrm{O}, \mathrm{O}-\mathrm{O}-\mathrm{Si}$, and $\mathrm{O}-\mathrm{O}-\mathrm{O}$ bond angle distributions (BADs). B The Si-Si-Si-Si, Si-O-Si-O and $\mathrm{O}-\mathrm{O}-\mathrm{O}-\mathrm{O}$ dihedral angle distributions, where several of the curves are offset vertically for clarity of presentation. In (A) and (B), the curves correspond to RT/7.7 GPa (black), $400^{\circ} \mathrm{C} / 7.7 \mathrm{GPa}$ (red), $1200^{\circ} \mathrm{C} / 7.7 \mathrm{GPa}$ (blue) and $\mathrm{RT} / 20 \mathrm{GPa}$ (cyan). The O-Si-O BADs have a peak at $\sim 109^{\circ}$ as expected for network structures built from tetrahedral $\mathrm{SiO}_{4}$ units. The $\mathrm{Si}-\mathrm{Si}-\mathrm{Si}$ BADs have a large peak at $\sim 109^{\circ}$ and a smaller feature at $\sim 60^{\circ}$ that originate from oxygen-decorated $\mathrm{SiSi}_{4}$ tetrahedra. Changes to the BADs are most noticeable with increasing density from RT/7.7 GPa to $400^{\circ} \mathrm{C} / 7.7 \mathrm{GPa}$ (e.g., there is a shift in position and broadening of the inter-tetrahedral Si-O-Si BAD) but are relatively subtle with a further increase in the density. 

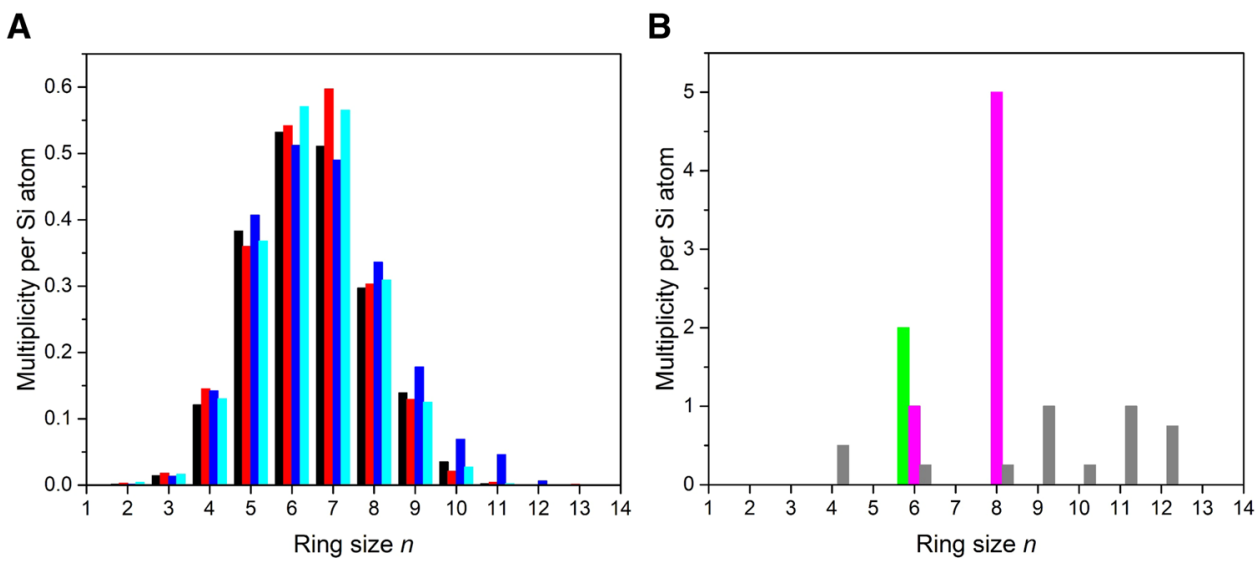

Fig. 6 Distribution of $\boldsymbol{n}$-fold primitive rings for glassy and crystalline $\mathrm{SiO}_{2}$. The rings were identified by making a shortest-path search, and $n$ is the number of $\mathrm{Si}$ atoms in a ring. In the analysis, duplicate rings are removed to avoid double counting. A The distributions obtained for $\mathrm{glassy} \mathrm{SiO}_{2}$ from the MD-RMC models: RT/7.7 GPa (black), $400^{\circ} \mathrm{C} / 7.7 \mathrm{GPa}$ (red), $1200^{\circ} \mathrm{C} / 7.7 \mathrm{GPa}$ (blue), and RT/20 GPa (cyan). B The distributions obtained from the crystal structures of a-cristobalite (green), a-quartz (magenta) and coesite (gray). For a-cristobalite, there is a single bar corresponding to sixfold rings; for a-quartz, there are two bars corresponding to sixfold and eightfold rings; and for coesite, there are seven bars corresponding to fourfold, sixfold, eightfold, ninefold, tenfold, elevenfold and twelvefold rings ${ }^{34}$.

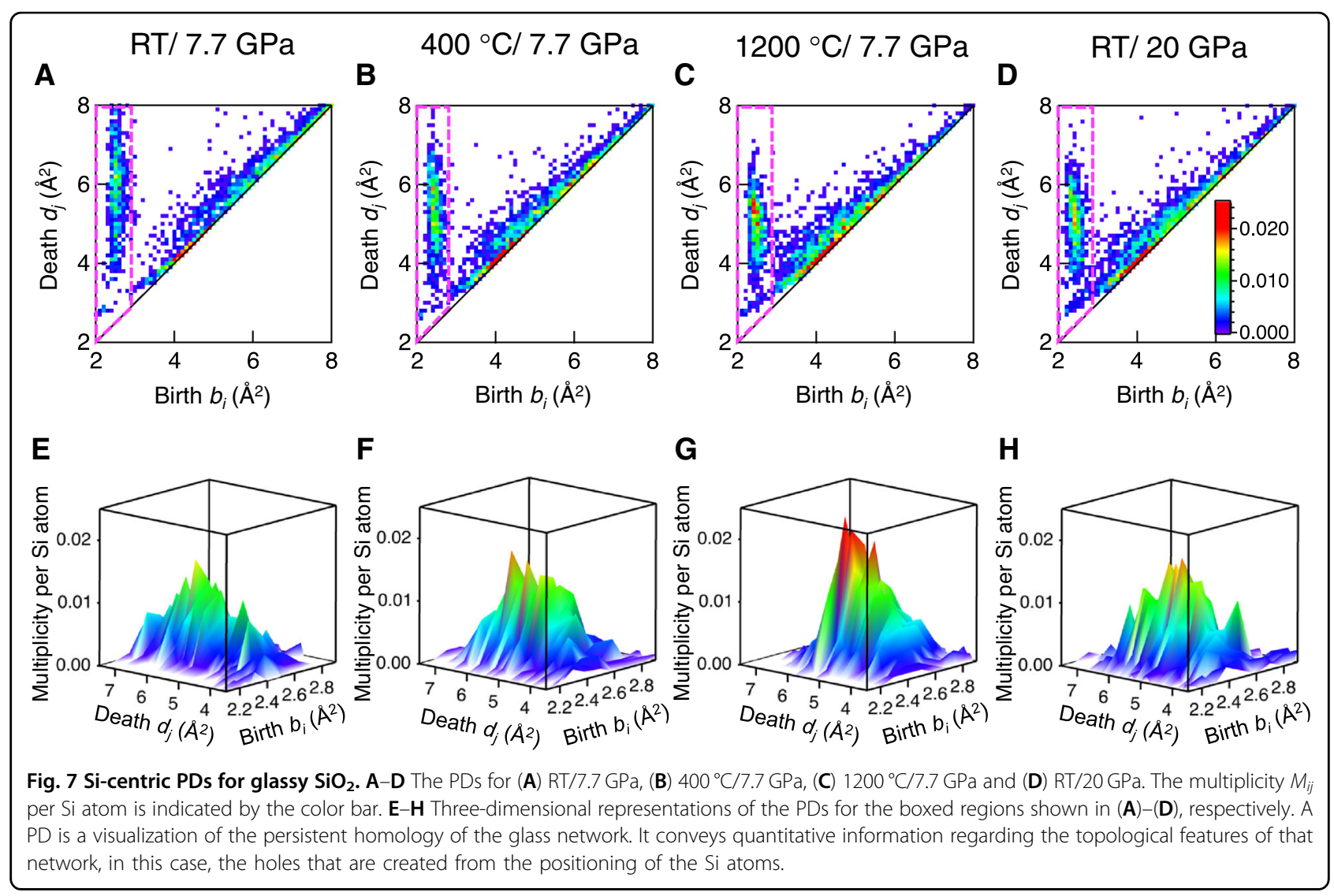

the challenge in producing sufficiently slow cooling rates that do not lead to the crystallization of a melt ${ }^{1,47,48}$.

Within the framework of a two-state model for silica, which can predict the anomalous density maximum of the liquid phase, LFTSs compete with more generic configurations as the state conditions are altered ${ }^{49}$. The LFTSs are identified with oxygen-decorated $\mathrm{SiSi}_{4}$ tetrahedra that dominate the FSDP in the Si-Si partial structure factor ${ }^{50}$. 

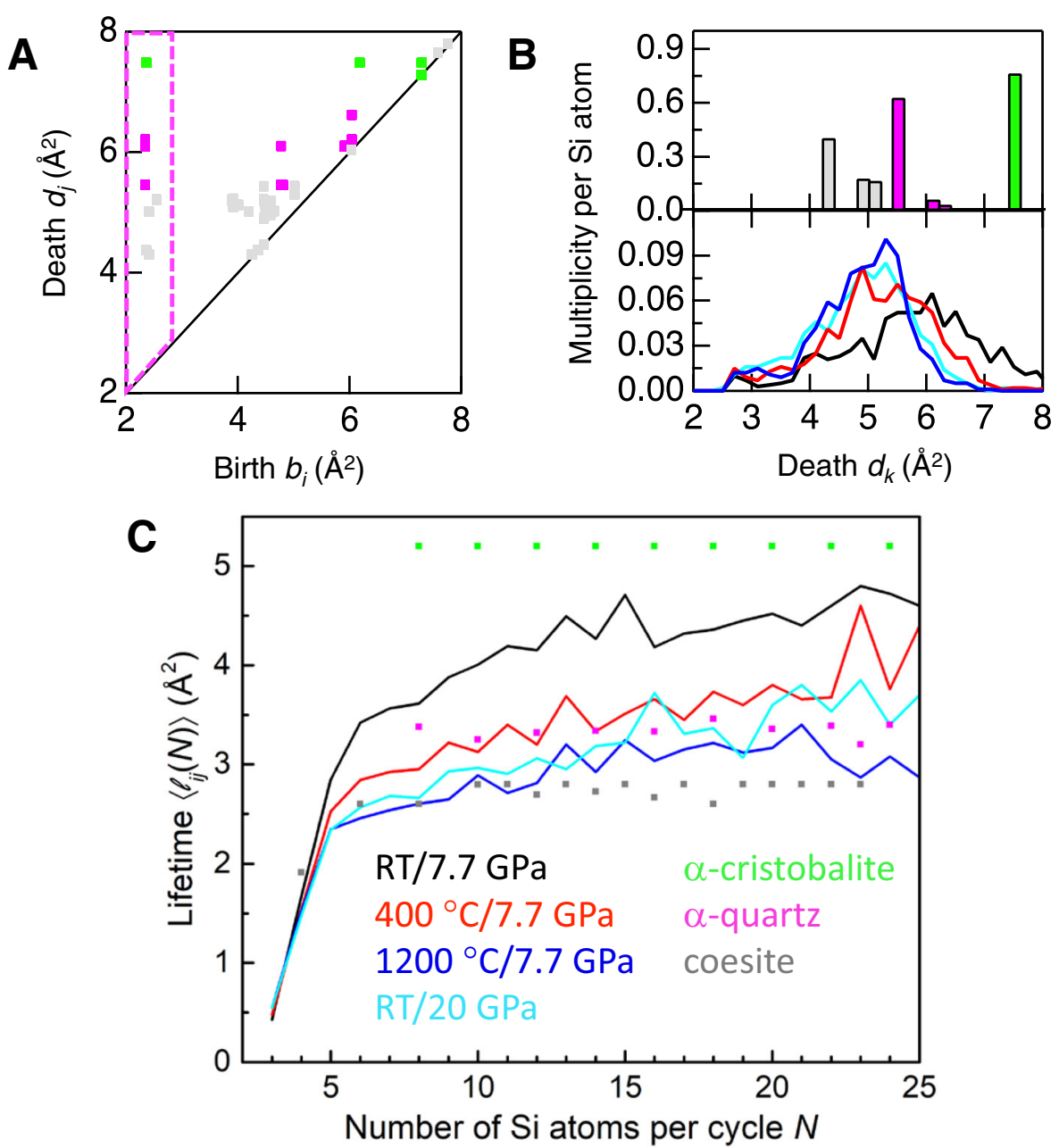

Fig. 8 Topology of glassy and crystalline $\mathrm{SiO}_{2}$ as described by $\mathbf{N S i}$ cycles. A The birth and death coordinates of the NSi cycles in the silica polymorphs. The symmetry of the crystalline state and the neglect of thermal broadening when constructing the PDs leads to a restricted set of coordinates relative to the glass. B The multiplicity profiles $\left\langle M_{i k}\right\rangle$ versus $d_{k}$ for the boxed regions in Figs. 8A and 7A-D, where the bars in the top panel represent the crystalline polymorphs and the curves in the bottom panel represent the densified glasses. The multiplicity is given by

$\left\langle M_{i k}\right\rangle=\sum_{i} M_{i k} / \sum_{i, j} M_{i j}$, where the summation in the numerator extends over all values of $b_{i}$ in the boxed region for a given value of $d_{k}$ and the summation in the denominator extends over all coordinates in the boxed region. It follows that $\sum_{k}\left\langle M_{i k}\right\rangle=1$ where the summation extends over all values of $d_{k}$ in the boxed region. C The average lifetime $\left\langle\ell_{i j}(N)\right\rangle$ of the persistent cycles found for each value of $N$ in the densified glasses (curves) and crystalline polymorphs (squares). The color scheme used to represent the various samples is shown in (C).

The states in the model are distinguished by using the translational order parameter $z \equiv r_{i j}-r_{i j}{ }^{\prime}$ where $r_{i j}{ }^{\prime}$ and $r_{i j}$ are the distances between $\mathrm{Si}$ atom $i$ and either its fourth nearest-neighbor $\mathrm{O}$ atom $j^{\prime}$ or its fifth nearestneighbor Si atom $j$, respectively. The LFTSs and other configurations are associated with larger versus smaller $z$ values, respectively; i.e., the order parameter gives a measure of the integrity of the LFTSs by monitoring the ingress of the second shell $\mathrm{Si}$ atoms. For hot-compressed glasses, the MD-RMC models show an increase in the $\mathrm{Si}-\mathrm{Si}$ coordination number with processing temperature, from 4.01 for $\mathrm{RT} / 7.7 \mathrm{GPa}$ to 4.17 for $1200^{\circ} \mathrm{C} /$
7.7 $\mathrm{GPa}$, which is accompanied by a decrease in the fraction of LFTSs from 95.5 to $83.4 \%$. Also, the $z$-parameter distribution develops a clear bimodal profile (Fig. 10A) and the LFTSs become less regular (Fig. 10B). These changes are associated with an initial decrease in the correlation length obtained from the FSDP, followed by a sharp increase (Fig. 1C). Hence, there appears to be a threshold concentration of non-LFTSs that is necessary to impart the glass network with a reorganizational flexibility.

Persistent homology provides an effective means for characterizing changes to the glass topology on multiple 
RT/ 7.7 GPa

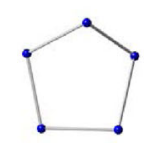

5 Si $(2.52,3.75)$

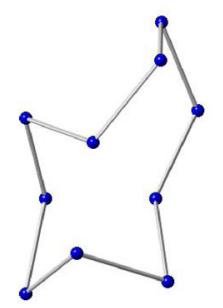

10Si $(2.50,4.19)$

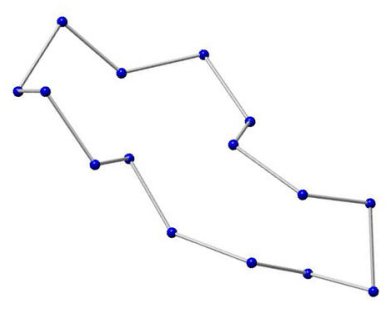

15Si $(2.51,3.83)$
$400{ }^{\circ} \mathrm{C} / 7.7 \mathrm{GPa}$

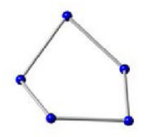

$5 \mathrm{Si}(2.50,2.79)$

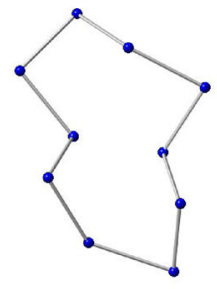

10Si $(2.42,2.83)$

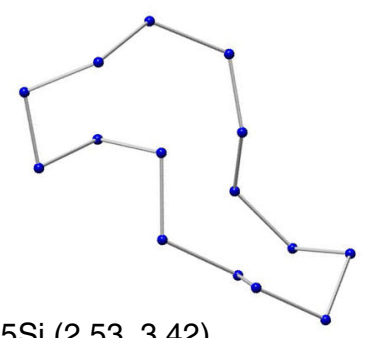

$15 \mathrm{Si}(2.53,3.42)$ $1200^{\circ} \mathrm{C} / 7.7 \mathrm{GPa}$

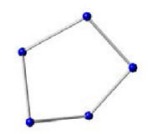

$5 \mathrm{Si}(2.50,2.75)$

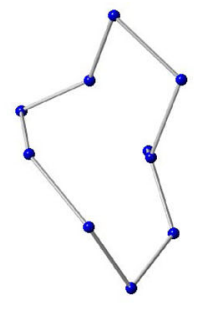

10Si $(2.44,2.23)$

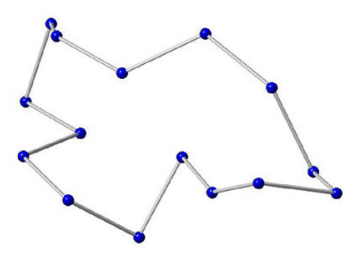

15Si (2.52, 3.22)
RT/ $20 \mathrm{GPa}$

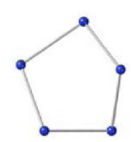

$5 \mathrm{Si}(2.38,2.45)$

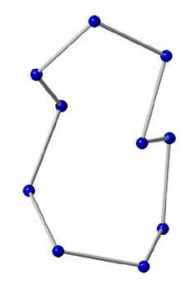

$10 \mathrm{Si}(2.45,3.20)$

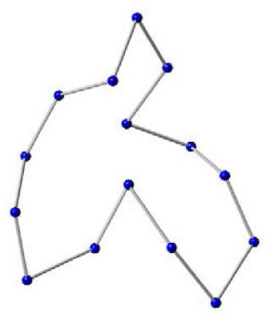

$15 \mathrm{Si}(2.45,2.85)$

Fig. 9 Several of the NSi cycles found by persistent homology for the compressed glasses. (Not to scale). The birth scale $b_{i}$ of a cycle corresponds to the radius at which the spheres, centered on each of the Si atom sites, first overlap to enclose a hole. The Si-Si distance between sites in a cycle $\leq 2 \sqrt{b_{i}}$, so each cycle is represented by Si atoms (solid circles) joined by bars of length $\leq 2 \sqrt{b_{i}}$. The death scale $d_{j}$ of a cycle corresponds to the radius at which the spheres cover the enclosed hole such that the hole is eliminated. The coordinates $\left(b_{i}, \ell_{i j}\right)$ give the birth and lifetime values of a cycle (in units of $\AA^{2}$ ) where $\ell_{i j}=d_{j}-b_{j}$. The cycles are optimal, i.e., each cycle corresponds to a minimal path length around a hole ${ }^{36}$.

length scales that are not highlighted by traditional characterization methods such as ring-size distributions. We have found that the growth in the correlation length for the glasses compressed at $7.7 \mathrm{GPa}$ (Fig. 1C) is associated with a preponderance of cycles associated with coesite-like death coordinates (Fig. 8B) and lifetime values (Fig. 8C). These findings do not suggest that the crystallization process has begun because the structure factors do not show Bragg peaks, and the primitive ring statistics do not show a shift towards the distribution expected for coesite (Fig. 6). The associated atomic arrangements may indicate, however, the likely precursors to crystal growth as viewed from the standpoint of the densified glass. Persistent homology offers the ability to seamlessly follow the glass-to-crystal transition, which is nontrivial given the broken symmetry in disordered materials, and should therefore be important for helping to understand the process of crystal nucleation ${ }^{2}$.

We have also used persistent homology to characterize the structural disorder in two same-density glasses that were prepared via the hot-compression versus coldcompression routes and found a contrast (Fig. 7G, H) that leads to different atomic dynamics (Fig. 3A, C) and thermodynamics (Fig. 3B, D). Although the boson peak in the low-frequency dynamical spectrum of silica glass does not originate solely from structural disorder because it is also present as a sharper feature in the spectrum for a same-density crystalline phase $\mathrm{e}^{20,21}$, our results reveal that the nature of this disorder does affect both the distribution of modes contributing towards the boson peak and the heat capacity of the glass. In glassy silica, the boson peak is likely to originate mostly from the oxygen motion associated with the libration and distortion of tetrahedral $\mathrm{SiO}_{4}$ motifs ${ }^{51,52}$. The present work shows these motifs to be organized differently on an intermediate length scale; i.e., the nature of the motion is sensitive to the connectivity of the tetrahedral units.

In summary, our approach uses persistent homology to characterize the geometry of atomic configurations in a 


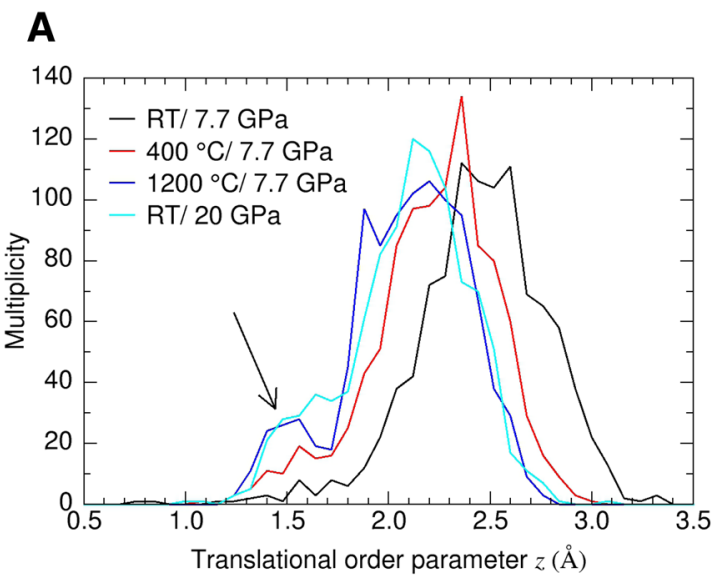

B

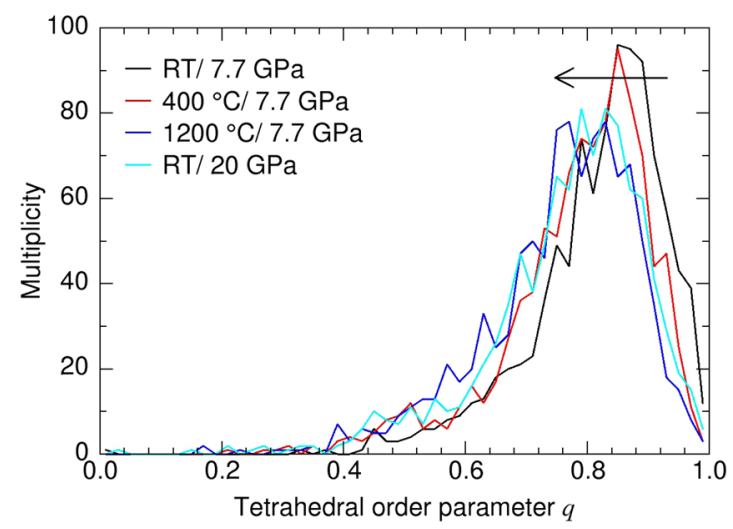

Fig. 10 Distributions of the translational and tetrahedral order parameters for the MD-RMC models of glassy $\mathrm{SiO}_{2}$. A The

z-parameter distribution for the glasses compacted at $7.7 \mathrm{GPa}$ develops a bimodal profile with increasing density as the LFTSS are replaced by other configurations indicated by the arrow. A bimodal distribution is also found for RT/20 GPa. B The peak in the q-parameter distribution moves in the direction of the arrow with increasing glass density as the LFTSs become increasingly distorted.

wide range of silica glasses that were engineered by using hot or cold compression. This method may therefore provide a missing key for decoding glass structures on multiple length scales that is helpful for the design of new glass or glass-ceramic materials with novel characteristics, where the design strategy is based on the knowledge of the atomic structure and how it influences the material properties.

\section{Acknowledgements}

Discussions with Profs. T. Otomo, K. Terakura, S. Tsuneyuki, M. Hatanaka and D. Hiench, and with Drs. K. Suzuya, M. Kobayashi, J. Hook and A. Souslov are gratefully acknowledged and appreciated. We thank Prof. A. Chumakov for providing the heat capacity data sets from Chumakov et al..$^{20}$. The synchrotron radiation experiments were performed with the approval of the Japan Synchrotron Radiation Research Institute (JASRI) (Proposal Nos. 2015A1366, 2016A0134, 2016B0134, 2017A0134, and 2017B0134). A portion of this research used resources at the Spallation Neutron Source, a DOE Office of Science User Facility operated by the Oak Ridge National Laboratory. This research was supported by JST PRESTO Grant Numbers JPMPR15N4 (to S. Kohara), JPMPR15ND (to T.N.), and JPMJPR16N6, Japan (to M.S.); the "Materials Research by Information Integration" initiative $\left(\mathrm{MI}^{2} \mathrm{I}\right)$ project of the Support Program for Starting Up Innovation Hub from JST (to Y.O., S. Kohara, A.M., S.T. and Y.H.); JST CREST 15656429 (to Y.H.); JSPS KAKENHI Grant Numbers JP17H03121 (to A.M.),

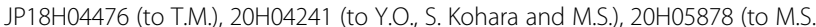
and S. Kohara), $20 \mathrm{H05880} \mathrm{(to} \mathrm{A.M.),} 20 \mathrm{H05881}$ (to Y.O., S. Kohara and A.H.), and $20 \mathrm{H} 05884$ (to M.S. and I.O.); and the TIA collaborative research program "Kakehashi", TK19-004 (to S. Kohara and T.M.). P.S.S. was supported by the Institute for Mathematical Innovation at the University of Bath, Grant No. IMI/ 201920/011. A.Z. was supported by a Royal Society - EPSRC Dorothy Hodgkin Research Fellowship. A.P. was supported by a PhD studentship funded by the Institut Laue Langevin (ILL) and University of Bath (Collaboration Agreement ILL-1353.1).

\section{Author details}

${ }^{1}$ Institute for Integrated Radiation and Nuclear Science, Kyoto University, 21010 Asashiro-nishi, Kumatori-cho, Sennan-gun, Osaka 590-0494, Japan. ${ }^{2}$ Center for Materials Research by Information Integration (CMI2), Research and Services Division of Materials Data and Integrated System (MaDIS), National Institute for Materials Science (NIMS), Ibaraki 305-0047, Japan. ${ }^{3}$ Research Center for Advanced Measurement and Characterization, NIMS, 1-1-1 Kouto, Sayo-cho, Sayo-gun, Hyogo 679-5148, Japan. ${ }^{4}$ PRESTO, Japan Science and Technology Agency, 7 Gobancho, Chiyoda-ku, Tokyo 102-0076, Japan. ${ }^{5}$ Diffraction and Scattering Division, Center for Synchrotron Radiation Research, Japan Synchrotron Radiation Research Institute, 1-1-1 Kouto, Sayo-cho, Sayo-gun, Hyogo 679-5198, Japan. ${ }^{6}$ Department of Physics, University of Bath, Bath BA2 7AY, UK. 'Department of Materials Science, Waseda University, 3-4-1 Ohkubo, Shinjuku, Tokyo 169-8555, Japan. ${ }^{8}$ Kagami Memorial Research Institute for Materials Science and Technology, Waseda University, 2-8-26 Nishiwaseda, Shinjuku, Tokyo 169-0051, Japan. ${ }^{9}$ Mathematics for Advanced Materials-OIL, AIST, 2-1-1 Katahira, Aoba-ku, Sendai 980-8577, Japan. ${ }^{10}$ WPI Advanced Institute for Materials Research, Tohoku University, 2-1-1 Katahira, Aoba-ku, Sendai 9808577, Japan. ${ }^{11}$ Laboratory for Materials and Structures, Tokyo Institute of Technology, 4259 Nagatsuta-cho, Midori-ku, Yokohama, Kanagawa 226-8503, Japan. ${ }^{12}$ Deutsches Elektronen-Synchrotron, Notkestrasse 85, 22607 Hamburg, Germany. ${ }^{13}$ Department of Electrical, Electronic and Computer Engineering, Faculty of Engineering, Gifu University, Gifu 501-1193, Japan. ${ }^{14}$ Center for Advanced Intelligence Project, RIKEN, 1-4-1 Nihonbashi, Chuo-ku, Tokyo 1030027, Japan. ${ }^{15}$ Graduate School of Science and Technology, Hirosaki University, 3 Bunkyo-cho, Hirosaki 036-8561, Japan. ${ }^{16}$ Institute of Industrial Science, The University of Tokyo, 4-6-1 Komaba, Meguro-ku, Tokyo 153-8505, Japan. ${ }^{17}$ Faculty of Science, University of the Ryukyus, 1 Senbaru, Nishihara-cho, Nakagami-gun, Okinawa 903-0213, Japan. ${ }^{18}$ Institut Laue-Langevin, 71 Avenue des Martyrs, F-38042, Grenoble, Cédex 9, France. ${ }^{19}$ Department of Materials Science, University of Tsukuba, 1-1-1 Tennodai, Tsukuba, Ibaraki 305-8573, Japan. ${ }^{20}$ Neutron Scattering Division, Oak Ridge National Laboratory, Oak Ridge, TN 37831, USA. ${ }^{21}$ Computer Science and Mathematics Division, Oak Ridge National Laboratory, Oak Ridge, TN 37831, USA. ${ }^{22}$ ISIS Facility, Rutherford Appleton Laboratory, Chilton, Didcot, Oxon OX11 0QX, UK. ${ }^{23}$ Kyoto University Institute for Advanced Study, WPI-ASHBi, Kyoto University, Yoshida Ushinomiya- cho, Sakyo-ku, Kyoto 606-8501, Japan. ${ }^{24}$ CREST, Japan Science and Technology Agency, 7 Gobancho, Chiyoda-ku, Tokyo 102-0076, Japan. ${ }^{25}$ Research Center for Computational Design of Advanced Functional Materials, National Institute of Advanced Industrial Science and Technology, 1-1-1 Umezono, Tsukuba, Ibaraki 305-8568, Japan. ${ }^{26}$ Department of Physics, Norwegian University of Science and Technology, NO, 7491 Trondheim, Norway. ${ }^{27}$ Computational Physics Laboratory, Tampere University, P. O. Box 692Fl-33014 Tampere, Finland. ${ }^{28}$ Department of Physical Sciences, Ritsumeikan University, 1-1-1 Nojihigashi, Kusatsu 525-8577, Japan. ${ }^{29}$ International Center for Materials Nanoarchitectonics, NIMS, 1-1 Namiki, Tsukuba 305-0044, Japan

\section{Author contributions}

S. Kohara and P.S.S. designed the research and wrote the paper. N.N. and T.T. synthesized the samples; Y.O., S. Kohara, A.M. and K.O. conducted the X-ray diffraction work; A.Z., A.P., H.E.F., P.S.S., Y.O., S. Kohara and A.C.H. carried out the neutron diffraction work; A.I.K. and M.B.S. performed the inelastic neutron scattering measurements; S. Kitani and H.K. conducted the specific heat measurements; and H.I. and S.T. performed the MD simulations. Y.O., A.H., S. Kojima, T.M., Y.H., I.O., E.G.S., M.G.T., M.T.M., T.N., J.A., Y.F., O.S., P.S.S., A.Z. and M.S. 
were involved in constructing the other atomistic models and in analyzing the configurations.

\section{Data availability}

Neutron diffraction data sets are available as S. Kohara, A.M., A.C.H., A.Z., Y.O., P. S.S., Structure of permanently densified $\mathrm{SiO}_{2}$ glass. ISIS Neutron and Muon Source, UK (2017) doi:10.5286/ISIS.E.RB1620365; S. Kohara, H.E.F., A.P., P.S.S., A.Z., Structure of $\mathrm{SiO}_{2}$ recovered from high pressure. Institut Laue-Langevin, Grenoble, France (2016) doi:10.5291/ILL-DATA.TEST-2596. The other data sets are available from the authors upon request.

\section{Conflict of interest}

The authors declare that they have no conflict of interest.

\section{Publisher's note}

Springer Nature remains neutral with regard to jurisdictional claims in published maps and institutional affiliations.

Supplementary information is available for this paper at https://doi.org/ 10.1038/s41427-020-00262-z

Received: 13 July 2020 Revised: 2 October 2020 Accepted: 8 October 2020. Published online: 23 December 2020

\section{References}

1. Greaves, G. N. \& Sen, S. Inorganic glasses, glass-forming liquids and amorphizing solids. Adv. Phys. 56, 1-116 (2007).

2. Zanotto, E. D., Tsuchida, J. E., Schneider, J. F. \& Eckert, H. Thirty-year quest for structure-nucleation relationships in oxide glasses. Int. Mater. Rev. 60, 376-391 (2015).

3. Salmon, P. S. In Magmas under Pressure (eds. Kono, Y. \& Sanloup, C.) Ch. 13, 343-369 (Elsevier, Amsterdam, 2018).

4. Lee, $\mathrm{S}$. K. et al. Oxygen quadclusters in $\mathrm{SiO}_{2}$ glass above megabar pressures up to 160 GPa revealed by x-ray Raman scattering. Phys. Rev. Lett. 123, 235701 (2019).

5. Lee, S. K. et al. Degree of permanent densification in oxide glasses upon extreme compression up to $24 \mathrm{GPa}$ at room temperature. J. Phys. Chem. Lett. 11, 2917-2924 (2020).

6. Guerette, M. et al. Structure and properties of silica glass densified in cold compression and hot compression. Sci. Rep. 5, 15343 (2015).

7. Salmon, P. S. \& Huang, L. Impact of pressure on the structure of glass and its material properties. MRS Bull. 42, 734-737 (2017).

8. Kapoor, S., Wondraczek, L. \& Smedskjaer, M. M. Pressure-induced densification of oxide glasses at the glass transition. Front. Mater. 4, 1 (2017).

9. McMillan, P., Piriou, B. \& Couty, R. A Raman study of pressure-densified vitreous silica. J. Chem. Phys. 81, 4234-4236 (1984).

10. Heaney, P. J. In Silica: Physical Behavior, Geochemistry and Materials Applications (eds. Heaney, P. J., Prewitt, C. T. \& Gibbs, G. V.) Ch. 1, 1-40 (Reviews in Mineralogy, Vol. 29, Mineralogical Society of America, Washington, D. C., 1994).

11. Inamura, Y., Katayama, Y., Utsumi, W. \& Funakoshi, K. Transformations in the intermediate-range structure of $\mathrm{SiO}_{2}$ glass under high pressure and temperature. Phys. Rev. Lett. 93, 015501 (2004).

12. Huang, L. \& Kieffer, J. Amorphous-amorphous transitions in silica glass. II. Irreversible transitions and densification limit. Phys. Rev. B 69, 224204 (2004).

13. Trease, N. M., Clark, T. M., Grandinetti, P. J., Stebbins, J. F. \& Sen, S. Bond lengthbond angle correlation in densified silica - Results from ${ }^{17} \mathrm{O}$ NMR spectroscopy. J. Chem. Phys. 146, 184505 (2017).

14. Zeidler, A. et al. High-pressure transformation of $\mathrm{SiO}_{2}$ glass from a tetrahedral to an octahedral network: a joint approach using neutron diffraction and molecular dynamics. Phys. Rev. Lett. 113, 135501 (2014).

15. Royall, C. P., Turci, F., Tatsumi, S. \& Russo, J. The race to the bottom: approaching the ideal glass? J. Phys.: Condens. Matter 30, 363001 (2018).

16. McGreevy, R. L. \& Pusztai, L. Reverse Monte Carlo simulation: a new technique for the determination of disordered structures. Mol. Simul. 1, 359-367 (1988).

17. Nakamura, T., Hiraoka, Y., Hirata, A., Escobar, E. G. \& Nishiura, Y. Persistent homology and many-body atomic structure for medium-range order in the glass. Nanotechnology 26, 304001 (2015).
18. Hiraoka, Y. et al. Hierarchical structures of amorphous solids characterized by persistent homology. Proc. Natl Acad. Sci. USA 113, 7035-7040 (2016).

19. Salmon, P. S., Martin, R. A., Mason, P. E. \& Cuello, G. J. Topological versus chemical ordering in network glasses at intermediate and extended length scales. Nature 435, 75-78 (2005).

20. Chumakov, A. I. et al. Role of disorder in the thermodynamics and atomic dynamics of glasses. Phys. Rev. Lett. 112, 025502 (2014).

21. Chumakov, A. I. \& Monaco, G. Understanding the atomic dynamics and thermodynamics of glasses: status and outlook. J. Non-Cryst. Solids 407, 126-132 (2015).

22. Kanke, Y., Akaishi, M., Yamaoka, S. \& Taniguchi, T. Heater cell for materials synthesis and crystal growth in the large volume high pressure apparatus at 10 GPa. Rev. Sci. Instrum. 73, 3268-3270 (2002).

23. Kohara, S. et al. Synchrotron x-ray scattering measurements of disordered materials. Z. Phys. Chem. 230, 339-368 (2016).

24. Hannon, A. C. Results on disordered materials from the GEneral Materials diffractometer, GEM, at ISIS. Nud. Instrum. Methods Phys. Res. A 551, 88-107 (2005).

25. Fischer, H. E. et al. D4c: a very high precision diffractometer for disordered materials. Appl. Phys. A 74, S160-S162 (2002).

26. Granroth, G. E. et al. SEQUOIA: a newly operating chopper spectrometer at the SNS. J. Phys.: Conf. Ser. 251, 012058 (2010).

27. Stone, M. B. et al. Comparison of four direct geometry time-of-flight spectrometers at the Spallation Neutron Source. Rev. Sci. Instrum. 85, 045113 (2014).

28. Arnold, O. et al. Mantid - Data analysis and visualization package for neutron scattering and $\mu S R$ experiments. Nucl. Instrum. Methods Phys. Res. A 764, 156-166 (2014).

29. Azuah, R. T. et al. DAVE: a comprehensive software suite for the reduction, visualization, and analysis of low energy neutron spectroscopic data. J. Res. Natl Inst. Stand. Technol. 114, 341-358 (2009).

30. Gereben, O., Jóvári, P., Temleitner, L. \& Pusztai, L. A new version of the RMC++ reverse Monte Carlo program, aimed at investigating the structure of covalent glasses. J. Optoelectron. Adv. Mater. 9, 3021-3027 (2007).

31. Heimbach, I. et al. pyMolDyn: identification, structure, and properties of cavities/vacancies in condensed matter and molecules. J. Comput. Chem. 38, 389-394 (2017).

32. Zeidler, A. et al. Structure of liquid and glassy $\mathrm{ZnCl}_{2}$. Phys. Rev. B 82, 104208 (2010).

33. Le Roux, S. \& Jund, P. Ring statistics analysis of topological networks: new approach and application to amorphous $\mathrm{GeS}_{2}$ and $\mathrm{SiO}_{2}$ systems. Comput. Mater. Sci. 49, 70-83 (2010). 50, 1217 (2011).

34. Marians, C. S. \& Hobbs, L. W. Network properties of crystalline polymorphs of silica. J. Non-Cryst. Solids 124, 242-253 (1990).

35. Obayashi, I. HomCloud. (Hiraoka Laboratory, Advanced Institute for Materials Research, Tohoku University, Japan, 2018). https://www.wpi-aimr.tohoku.ac.jp/ hiraoka_labo/homcloud/index.en.html.

36. Obayashi, I. Volume-optimal cycle: tightest representative cycle of a generator in persistent homology. SIAM J. Appl. Algebra Geom. 2, 508-534 (2018).

37. Errington, J. R. \& Debenedetti, P. G. Relationship between structural order and the anomalies of liquid water. Nature 409, 318-321 (2001).

38. Salmon, P. S. Real space manifestation of the first sharp diffraction peak in the structure factor of liquid and glassy materials. Proc. R. Soc. Lond. A $\mathbf{4 4 5}$ 351-365 (1994).

39. Sokolov, A. P., Kisliuk, A., Soltwisch, M. \& Quitmann, D. Medium-range order in glasses: comparison of Raman and diffraction measurements. Phys. Rev. Lett. 69, 1540-1543 (1992).

40. Sugai, S. \& Onodera, A. Medium-range order in permanently densified $\mathrm{SiO}_{2}$ and $\mathrm{GeO}_{2}$ glass. Phys. Rev. Lett. 77, 4210-4213 (1996).

41. Inamura, $Y$. et al. Intermediate range structure and low-energy dynamics of densified vitreous silica. J. Non-Cryst. Solids 293-295, 389-393 (2001).

42. Price, D. L. \& Carpenter, J. M. Scattering function of vitreous silica. J. Non-Cryst. Solids 92, 153-174 (1987).

43. Whittaker, D. A. J. et al. Partial vibrational density of states for amorphous solids from inelastic neutron scattering. Phys. Rev. B 98, 064205 (2018).

44. Pohl, R. O. in Amorphous Solids: Low-Temperature Properties (ed. Phillips, W. A.) Ch 3, 27-52 (Topics in Current Physics Vol. 24, Springer, Berlin, 1981).

45. Salmon, P. S. \& Zeidler, A. Ordering on different length scales in liquid and amorphous materials. J. Stat. Mech. 114006 (2019).

46. Angell, C. A. Formation of glasses from liquids and biopolymers. Science $\mathbf{2 6 7}$ 1924-1935 (1995). 
47. Machon, D., Meersman, F., Wilding, M. C., Wilson, M. \& McMillan, P. F. Pressureinduced amorphization and polyamorphism: Inorganic and biochemical systems. Prog. Mater. Sci. 61, 216-282 (2014).

48. Greaves, G. N. et al. The rheology of collapsing zeolites amorphized by temperature and pressure. Nat. Mater. 2, 622-629 (2003).

49. Shi, R. \& Tanaka, H. Impact of local symmetry breaking on the physical properties of tetrahedral liquids. Proc. Natl Acad. Sci. USA 115, 1980-1985 (2018).
50. Shi, R. \& Tanaka, H. Distinct signature of local tetrahedral ordering in the scattering function of covalent liquids and glasses. Sci. Adv. 5, eaav3194 (2019).

51. Buchenau, U., Nücker, N. \& Dianoux, A. J. Neutron scattering study of the low-frequency vibrations in vitreous silica. Phys. Rev. Lett. 53, 2316-2319 (1984).

52. Wehinger, B. et al. Lattice dynamics of a-cristobalite and the boson peak in silica glass. J. Phys.: Condens. Matter 27, 305401 (2015). 